\title{
NEGÓCIO BASEADO EM PROJETOS: O CASO DO INSTITUTO INTERNACIONAL DE FÍSICA
}

\section{PROJECT-BASED BUSINESS: THE CASE OF THE INTERNATIONAL PHYSICS INSTITUTE}

Recebido em: 07 maio 2020

Aprovado em: 19 set. 2020

Versão do autor aceita publicada online: 19 set. 2020

Publicado online: 04 jun. 2021

\section{Como citar esse artigo - American Psychological Association (APA):}

Brito, J. V. da C. S., Ribeiro, W. D., Silva, R. R., Medeiros Júnior, J. V., \& Sousa Neto, M. V. (2022, abr./jun.). Negócio baseado em projetos: o caso do Instituto Internacional de Física. Exacta. 20(2), 336-355. https://doi.org/10.5585/exactaep.2021.17163.

Submeta seu artigo para este periódico $७$

Dados Crossmark 


\section{NEGÓCIO BASEADO EM PROJETOS: O CASO DO INSTITUTO INTERNACIONAL DE FÍSICA}

\section{PROJECT-BASED BUSINESS: THE CASE OF THE INTERNATIONAL PHYSICS INSTITUTE}

\author{
iD Jéssica Vivianne da Cunha Silva de Brito \\ iD Waldelino Duarte Ribeiro ${ }^{2}$ \\ iD Rafael Rodrigues da Silva ${ }^{3}$ \\ iD Josué Vitor de Medeiros Júnior ${ }^{4}$ \\ iD Manoel Veras de Sousa Neto ${ }^{5}$
}

${ }^{1}$ Doutoranda em Administração

Universidade Federal do Rio Grande do Norte - UFRN brito.jessicacunha@gmail.com

${ }^{2}$ Mestrando em Ciência, Tecnologia e Inovação Universidade Federal do Rio Grande do Norte - UFRN waldelino.ribeiro@ufrn.br

${ }^{3}$ Doutor em Administração Instituto Federal de Educação, Ciência e Tecnologia do Rio Grande do Norte - IFRN rafaelrodges@outlook.com

${ }^{4}$ Doutor em Administração

Universidade Federal do Rio Grande do Norte - UFRN josuevitor16@gmail.com

${ }^{5}$ Doutor em Administração

Universidade Federal do Rio Grande do Norte - UFRN manoel.veras@uol.com.br

Recebido em: 07 maio 2020

Aprovado em: 19 set. 2020
Resumo: Este trabalho objetiva apresentar o processo de elaboração de planejamento estratégico do Instituto Internacional de Física (IIF) da Universidade Federal do Rio Grande do Norte (UFRN). Um aspecto inovador foi que o planejamento estratégico construído foi todo baseado no conceito de Negócio Baseado em Projetos (NBP), proposto por Veras (2017). O estudo desenvolveu-se sob uma perspectiva qualitativa com uso da observação participante. Verificou-se que a utilização do conceito de NBP possibilitou formular os projetos a partir do desdobramento dos objetivos estratégicos, com critérios de priorização e seleção, além de ter promovido o engajamento de todas as partes envolvidas na elaboração do planejamento. Este estudo contribui com a teoria à medida que desenvolve o conceito de NBP, o qual é pouco abordado na literatura nacional, e mostra a adoção prática de algumas ferramentas de gestão.

Palavras-chave: Planejamento estratégico. Negócio baseado em projetos. Gestão de projetos.

Abstract: This work aims to present the strategic planning process of the International Institute of Physics (IIF) of the Federal University of Rio Grande do Norte (UFRN). An innovative aspect was that strategic planning was based on the concept of Projectsbased Business (NBP), proposed by Veras (2017). The work was developed under a qualitative perspective using participant observation. It was verified that the use of the concept of NBP made it possible to formulate the projects from the unfolding of the strategic objectives, with criteria of prioritization and selection, besides having promoted the involvement of all the parties involved in the elaboration of the planning. This study contributes with a theory as it develops the concept of NBP, which is rarely addressed in the national literature, and shows the practice of some management tools.

Keywords: Strategic planning. Project-based businesses. Project management. 


\section{Introdução}

A implementação do planejamento estratégico é um dos maiores desafios que as organizações que querem se manter competitivas enfrentam (Mintzberg, 2004; Hill \& Jones, 2009). Sabe-se que cerca de $70 \%$ das estratégias corporativas não são implementadas e somente $60 \%$ do valor das estratégias são percebidas pelas organizações (Johnson, 2004). Assim, as organizações têm utilizado a gestão de projetos como um meio para implementação de estratégias ou do planejamento estratégico (Jamieson \& Morris, 2007; Kwak \& Anbari, 2009).

Todo planejamento estratégico é elaborado visando o sucesso no intuito de transformar a visão de futuro em realidade (Serra \& Kunc, 2015). Portanto, é necessário que os atores responsáveis pela gestão das organizações ou mesmo pelo processo de desenvolvimento de uma nova perspectiva estratégica façam uso de ferramentas, modelos e instrumentos teórico-metodológicos a fim de facilitar a implementação do planejamento e, por consequência, da estratégia organizacional de forma completa e eficaz.

No artigo seminal de Lord (1993), verificou-se que as técnicas de gestão de projetos forneceram uma estrutura adequada para o gerente monitorar o progresso de implementação do planejamento estratégico sem necessariamente usar a autoridade hierárquica. Em outro estudo, Cocks (2010) observou que a aplicação de técnicas de gestão de projetos foi um fator importante e ajudou na implementação de planos estratégicos em vários tipos de organizações. Alsudiri, Al-Karaghouli e Eldabi (2013) também observaram que a estratégia do negócio e a gestão de projetos precisam estar bem alinhadas para garantir um resultado bem-sucedido do projeto. Além desses, a literatura apresenta outros estudos que utilizaram a gestão de projetos como ferramenta para implementação da estratégia, por exemplo: Dietrich e Lehtonen (2005); Jamieson e Morris (2007); Meskendahl (2010); Guedes, Fonseca, Carvalho, Maximiniano e Gonçalves (2011); Musawir, Serra, Zwikael e Ali. (2017).

Nessa perspectiva, Veras (2017) apresentou um conceito para promover o alinhamento entre os objetivos estratégicos e a gestão de projetos chamado Negócio Baseado em Projeto (NBP). Segundo Veras (2017), negócios podem se utilizar da comercialização e execução de projetos como forma principal de criar valor para os clientes. Esse conceito permite maior alinhamento estratégico, pois sugere formular e implementar o planejamento estratégico por meio de projetos, ou seja, a ideia é que os objetivos estratégicos relacionados ao planejamento estratégico sejam movimentados por um ou mais projetos estratégicos associados a ele (Veras, 2017).

Nesse contexto, este trabalho objetiva apresentar a processo de elaboração de planejamento estratégico do Instituto Internacional de Física (IIF) da Universidade Federal do Rio Grande do Norte (UFRN) em que o planejamento foi baseado no conceito de NBP, proposto por Veras (2017). Esta pesquisa foi motivada pela necessidade de observar empiricamente a aplicação desse conceito, visando 
conhecer sua efetividade prática enquanto metodologia de suporte à elaboração do planejamento estratégico.

O lócus do estudo é o IIF, o qual é um instituto de pesquisa com vocação internacional, permanentemente voltado para as áreas de fronteira da física teórica. Desse modo, o planejamento estratégico elaborado para o Instituto foi estruturado em objetivos estratégicos alinhados a projetos, o que o conduziu a um modelo de negócio baseado em projetos.

Considerando que o conceito de NBP é recente, não foram identificados estudos que relacionaram este conceito à gestão de projetos e ao planejamento estratégico. Desse modo, este trabalho contribui para o entendimento sobre a relação entre as práticas de gerenciamento de projetos e a implementação de estratégias organizacionais baseando o negócio todo por projetos.

Este trabalho é composto por esta introdução, seguida pela discussão dos principais conceitos sobre planejamento estratégico, gestão de projetos e negócio baseado em projetos. Por conseguinte, são descritos os procedimentos e métodos adotados, bem como apresentado o processo de construção do planejamento estratégico do IIF. Por fim, são feitas as considerações finais e listadas as referências utilizadas.

\section{Fundamentação teórica}

\subsection{Planejamento estratégico e gestão de projetos}

A estratégia é a criação de uma posição única e valiosa, e que envolve um conjunto diferente de atividades. Também é definida como uma ação de fazer escolhas, executar atividades em um formato diferente de seus concorrentes para uma proposição de valor (Porter, 1996).

Veras (2017) define que a formulação da estratégia visa definir os caminhos que a organização deve trilhar para que sua visão de futuro se torne realidade. Conceito similar ao proposto por Freitas (2016, p. 42), que define uma visão estratégica em projetos como "definição de uma série de ações, olhando para o futuro, levando em conta a realidade, a necessidade e os desejos de uma organização e o conhecimento do gerenciamento de projetos por parte dos profissionais que o aplicam".

Tal perspectiva leva a compreensão de que o primeiro passo para que ocorra o alinhamento entre os projetos estratégicos e os objetivos organizacionais é a realização de um planejamento que traduza em itens específicos o que a organização almeja. Essa iniciativa é o principal fator que irá determinar o investimento ou não da organização em um projeto (Project Management Institute - PMI, 2017). Já o planejamento é o processo que, mediante a identificação das forças que exercem influências sobre o ambiente organizacional, define os caminhos que a organização deve percorrer para alcançar sua visão de futuro (Veras, 2017). Nesse sentido, Veras (2017), divide o planejamento estratégico em duas grandes fases, como mostra a Figura 1. 


\section{Figura 1}

Framework do planejamento estratégico

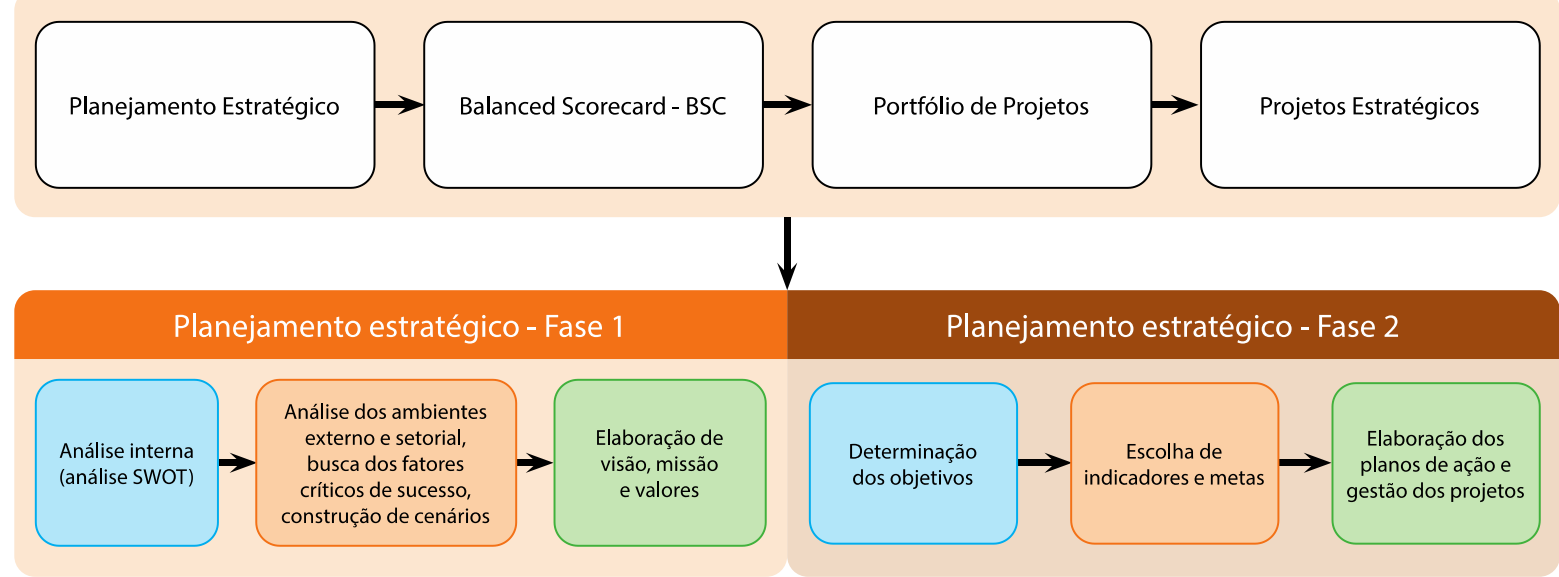

Fonte: Adaptado de Veras (2017).

De modo geral, a execução da estratégia organizacional mediante a gestão de projetos já foi abordada em outros estudos. Guedes et al. (2011), observaram que a chave para o alinhamento dos projetos com a estratégia organizacional é a gestão do portfólio de projetos, essa função seria a responsável por fazer convergir os esforços temporários ao propósito da organização. Já o estudo de Meskendahl (2010) indicou que a coordenação bem-sucedida do portfólio de projetos suscita maiores benefícios para a organização, isto é, gera importantes avanços para a organização em termos do alcance de seus objetivos.

Entende-se que é possível que um projeto seja um sucesso do ponto de vista dos objetivos, mas seja um fracasso no tocante às expectativas organizacionais. Nesse sentido, observa-se que não sendo possivel que um projeto contribua simultaneamente com ambos os objetivos, o ideal seria que ele possibilitasse de forma prioritária o alcance das metas organizacionais (Melton, lles-Smith, \& Yates, 2008)

De modo geral, as organizações precisam garantir o sucesso de seus projetos como um meio para alcançar êxito na execução das estratégias (Serra \& Kunc, 2015), mas o sucesso dos projetos deve ser medido em termos de sua contribuição para a organização (Dietrich \& Lehtonen, 2005).

A partir de uma revisão sistemática da literatura, Silva, Nascimento e Araújo (2017) observaram que o processo de implementação de estratégias pode ser suportado pela gestão de projetos. Os achados indicaram que a gestão de projetos (i) fornece estrutura para monitoramento e controle da implementação de estratégias, (ii) fornece estrutura de comunicação e feedback ao processo de implementação e planejamento estratégico, (iii) indica o alinhamento e a integração do gerenciamento de projetos com o processo de formulação da estratégia como um fator crítico de sucesso, (iv) indica 
que as habilidades do gerente e da equipe do projeto são um fator crítico de sucesso na implementação da estratégia, e (v) a existência de um Escritório de Gerenciamento de Projetos (EGP) contribui para implementação.

Veras (2017), por sua vez, discute acerca da integração dos projetos às metas da organização, na direção de que os projetos são meios que permitem alcançar as metas delineadas no planejamento estratégico da organização e devem estar associados à execução das ações definidas pelos objetivos estratégicos.

Conforme Melton, lles-Smith e Yates (2008), projetos executados no âmbito das organizações devem ser revisados continuamente quanto à sua viabilidade, se existem recursos e capacidades para desenvolvê-los, quanto à sua aceitabilidade, se eles se encaixam nas perspectivas dos stakeholders, e, principalmente, quanto à sua adequação, no tocante à direção da visão da organização. Nesse sentido, é relevante conceber as ações referentes à gestão de projetos como algo intrínseco à execução da estratégia, melhor dizendo, é necessário que os projetos executados sejam desenvolvidos sob metodologias eficazes que Ihes possibilitem gerar para a organização ganhos em termos de desempenho, o qual é entendido - neste estudo - como um esforço empreendido na direção dos resultados esperados, Veras (2017).

No âmbito da gestão de projetos, cabe à gestão do portfólio de projetos fazer a priorização dos projetos a serem executados de acordo com a estratégia e os objetivos da organização de modo a maximizar o valor dos projetos frente ao planejamento estratégico (Guedes et al., 2011; Young et al., 2012; PMI, 2017).

Para Dietrich e Lehtonen (2005), o processo de execução de uma estratégia organizacional é algo complexo e dinâmico por natureza, além de ser altamente influenciado por mudanças externas e também ser bastante abstrato. Hrebiniak (2006) complementa ao afirmar que é mais complicado fazer o trabalho de estratégia do que formular a estratégia. Nesse sentido, a gestão de portfólio não deve se restringir apenas a coordenação dos projetos, mas desempenhar o papel de facilitadora desse processo, exercendo de forma contínua o monitoramento quanto ao alinhamento dos projetos a serem executados ou em execução com as expectativas da organização.

Neste trabalho, a estratégia organizacional é analisada sob a perspectiva inovadora do conceito de NBPs, o qual pode ser compreendido como uma metodologia de planejamento estratégico que preconiza a integração dos objetivos institucionais aos projetos por meio de uma série de procedimentos, técnicas e artefatos. Assim, a próxima seção explica como a metodologia pode ser compreendida do ponto de vista de uma organização. Tais aspectos também são descritos na aplicação prática no processo de construção de um planejamento estratégico apresentados na quarta seção de Resultados. 


\subsection{Negócio Baseado em Projetos}

Considerando o dinamismo do mercado atual, os negócios precisam ser reconfigurados porque as antigas práticas de gestão, baseadas na burocracia, já não atendem mais às necessidades dos negócios. Para atender a determinadas demandas, os negócios precisam ser pensados por projeto, alocando, também, as pessoas e suas competências por projetos (Veras, 2017).

Duas definições se destacam quando se trata do conceito de Negócio Baseado em Projetos (NBP). A primeira se refere àquelas organizações cujo fluxo do negócio depende essencialmente da venda de projetos, se encaixando nessa perspectiva, por exemplo, empresas de arquitetura e engenharia, de consultoria, de sistemas de informação. A segunda, a qual adota-se como fundamento deste trabalho, diz respeito às entidades que se estruturam a partir de projetos, sejam eles de caráter interno ou externo, nesse caso a opção pela base em projetos não seria uma decorrência das características do negócio, mas uma escolha estratégica de estruturação organizacional (Anselmo \& Maximiano, 2011).

Um NBP é um negócio que opera por projetos, sendo o ciclo de vida do negócio o próprio ciclo de vida do projeto (Veras, 2017). Isto é, a cada projeto encerrado, finaliza-se um ciclo do negócio, e a cada projeto iniciado, estabelece-se um novo. Esse modelo de negócio deve ter sua estrutura fundamentada em práticas de gestão de projetos, as quais iriam nortear o negócio em todas as dimensões, conforme a Figura 2.

Tais dimensões facilitam a compreensão da estrutura organizacional baseada em projetos à medida que aloca os papéis da gestão de projetos nos níveis da hierarquia organizacional. Nesse sentido, cada uma dessas dimensões tem um propósito específico relacionado aos projetos, sendo: na dimensão estratégica, a determinação de quais projetos serão executados; na organizacional, a definição da forma como os projetos serão executados; e na operacional, a execução dos projetos de maneira efetiva, mediante a aplicação de ferramentas e técnicas de gestão de projetos (Anselmo \& Maximiano, 2011).

De modo geral, NBP são aqueles cuja execução de projetos Ihes possibilita buscar seus objetivos organizacionais, sendo os projetos, nessa visão, a ponte entre o planejamento estratégico e os resultados esperados com sua implementação. Como forma de definir mais adequadamente tais organizações pontua-se as seguintes características (Vargas, 2005):

- Os atores organizacionais têm disponibilidade para atuar (e gerenciar) projetos;

- O responsável pelo projeto, em geral o gerente do projeto, possui autoridade - e, algumas vezes, controle funcional - sobre os membros da equipe;

- A maior parte dos funcionários da organização integra algum projeto; 
- A organização destina recursos para treinamento e capacitação em práticas de gestão de projetos;

- A necessidade de apoio externo (consultorias) para a gestão dos projetos é algo incomum.

Figura 2

Dimensões do Negócio Baseado em Projetos (NBP)

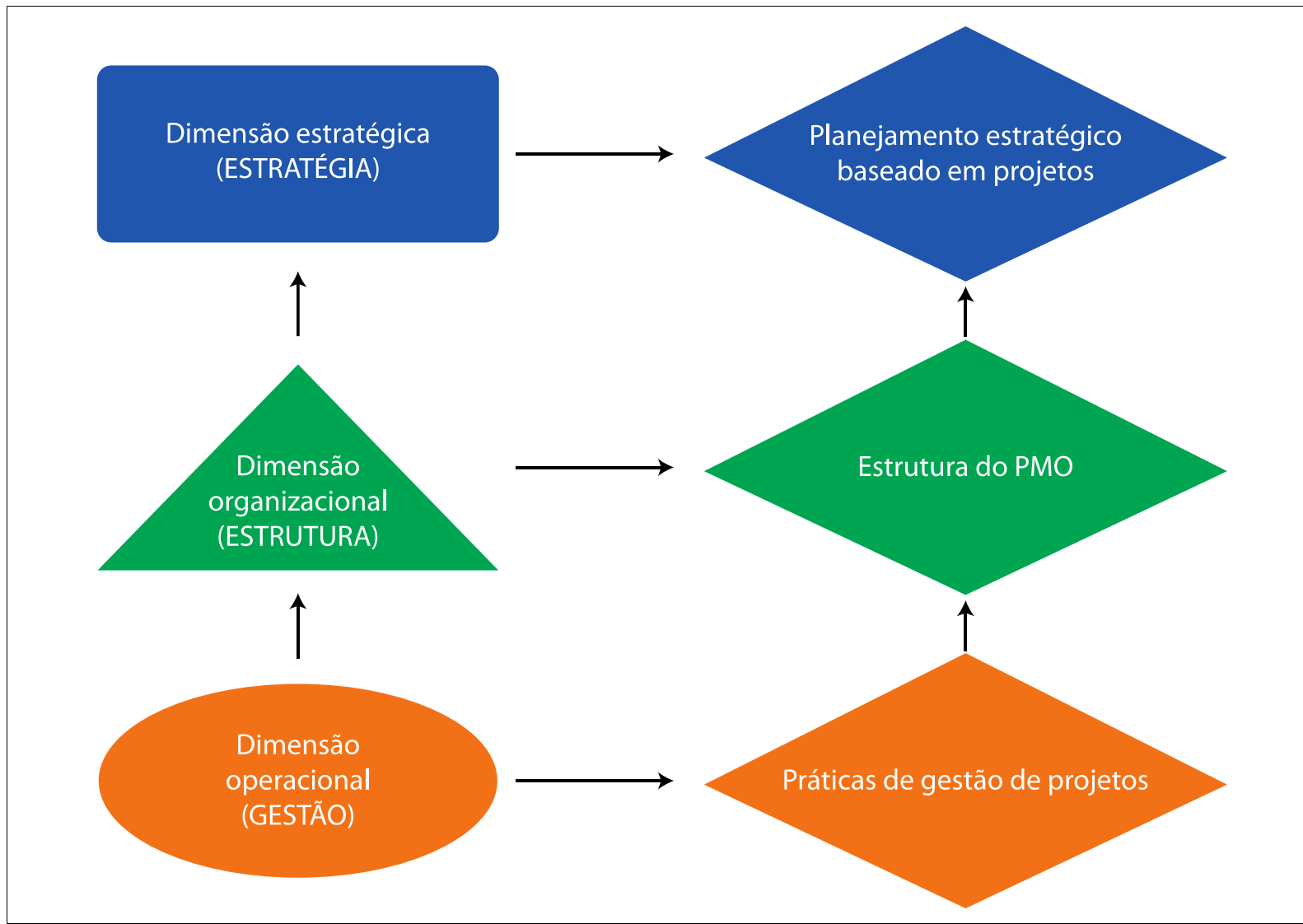

Fonte: Adaptado de Veras (2017, p. 9).

Veras (2017) resume o modelo de NBP a partir de um ciclo de melhoria contínua, o qual é constituído por cinco pontos que dão sustentação à inserção dos projetos como base de uma organização, quais sejam: "escolhas estratégicas", gestão de portfólio de projetos, escritório de projetos, gestão dinâmica de projetos e melhoria da maturidade da gestão de projetos. Um negócio baseado em projetos deve ter uma estrutura que vincule o planejamento estratégico a projetos e que apoie a execução desses projetos, além de uma configuração padrão que oriente o gerenciamento de tais projetos. Desse modo, Veras (2017) também sugere que o modelo de gestão visual de projetos Life Cycle Canvas (LCC) seja adotado como padrão para a gestão dos projetos estratégicos.

Silva Filho, Silva, Silva e Medeiros (2018) também sugeriram a associação do LCC com o Business Model Canvas (BMC), considerando o processo empreendedor. A apresentação das ideias indicou que 
há complementaridade entre as duas ferramentas, sendo o BMC responsável pela fase inicial do processo empreendedor e o LCC pela fase final de gestão dos projetos que permitiram a continuidade e sucesso do negócio.

O LCC, permite operacionalizar a estratégia do negócio possibilitando gerenciar todo as fases do ciclo de vida do projeto: iniciação; planejamento; execução e monitoramento; e encerramento. Em cada fase do ciclo de vida do projeto é possível gerar artefatos, utilizando-se de técnicas e ferramentas distintas (Veras, 2017). A Figura 3 ilustra a tela principal do LCC.

Figura 3

Tela do Life Cycle Canvas

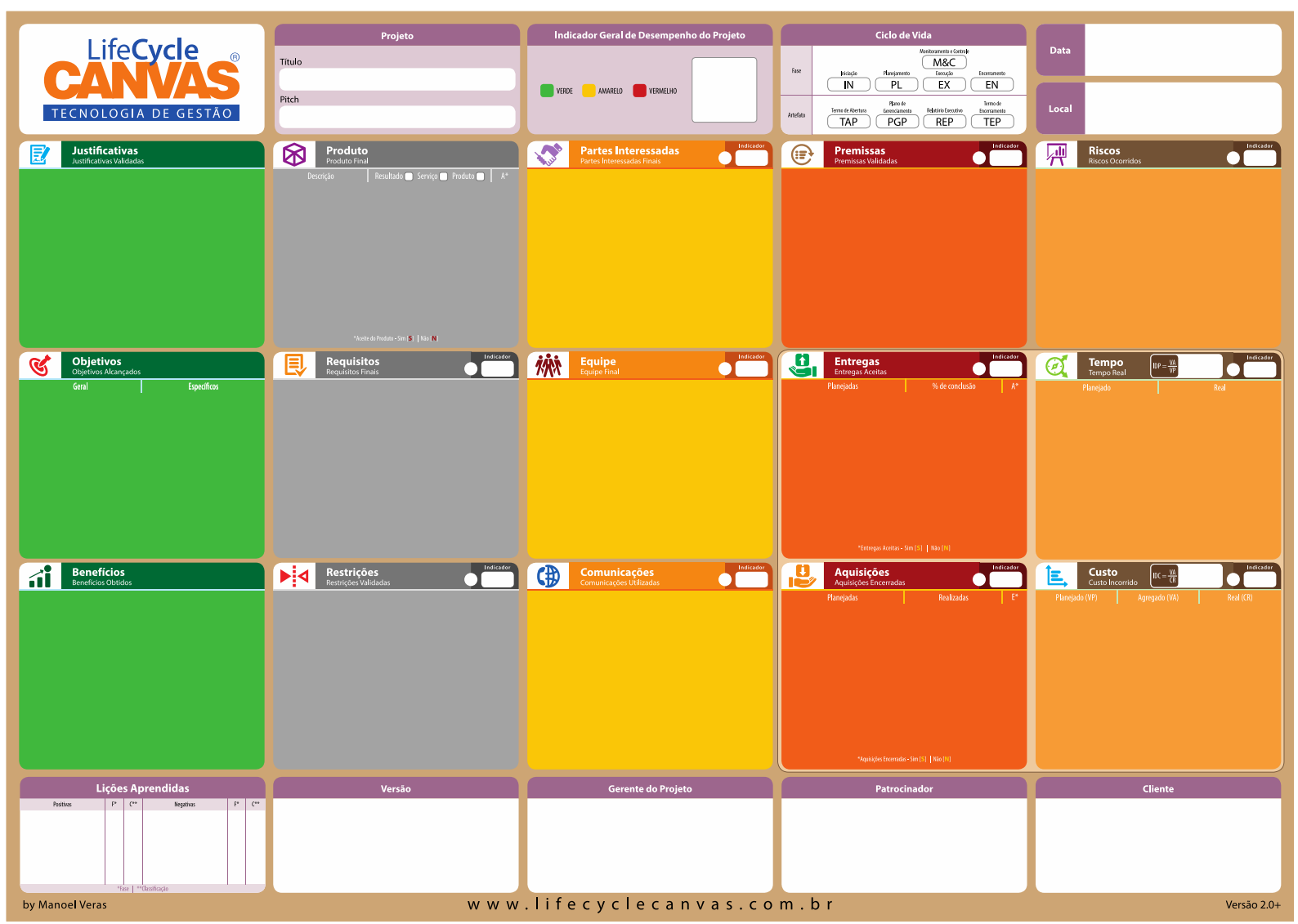

Fonte: Adaptado de Veras (2017, p. 103).

De acordo com Veras e Campelo (2017), o LCC torna a Gestão de Projetos mais simples, intuitiva e dinâmica. Construída através de uma base conceitual forte, alinhada ao Guia PMBOK, também é aderente à metodologia PRINCE2 e a metodologias ágeis. Dessa maneira, o LCC responde a questões importantes relacionadas ao projeto, e que ajudam no alcance do sucesso (Veras \& Campelo, 2017). 


\section{Procedimentos e métodos da pesquisa}

Este artigo é classificado, quanto à abordagem, como uma pesquisa qualitativa básica. Conforme Flick (2009), a abordagem qualitativa busca descrever e às vezes explicar os fenômenos sociais, analisando as experiências de indivíduos ou grupos e tentando compreender como as pessoas constroem e atribuem sentido ao mundo à sua volta. No caso desta pesquisa, busca-se demonstrar como foi desenvolvido o processo de elaboração do planejamento estratégico utilizando o conceito de NBP.

Quanto aos fins, trata-se de uma investigação descritiva, pois essa finalidade é adequada quando se busca estabelecer um perfil preciso de algum evento ou situação (Saunders, Lewis \& Thornhill, 2016). A estratégia de pesquisa utilizada foi a estudo de caso do tipo instrumental. Esse método é indicado quando se estuda um caso que, apesar de singular, tem um alto potencial de aplicabilidade em outras situações similares (Yin, 2016).

A coleta dos dados foi realizada por meio de observação participante entre os meses de julho e dezembro de 2018. Nessa forma de coleta o observador geralmente está envolvido naquilo que ele está observando, sendo uma das ferramentas-chave para a coleta dos dados em pesquisa qualitativa (Creswell, 2014). Durante a coleta foram realizadas anotações sobre o processo observado e levantados documentos elaborados durante a construção do planejamento estratégico objeto deste trabalho.

No que diz respeito ao envolvimento, pontua-se que um dos autores deste trabalho participou ativamente de todo processo de elaboração do planejamento estratégico, o que também se caracteriza como uma observação com "participante completo", onde o pesquisador está totalmente envolvido com o que está sendo observado (Creswell, 2014). Ao todo as atividades de observação foram realizadas em 58 reuniões, que envolveram 98 horas de trabalho, aproximadamente.

Considerando os procedimentos éticos propostos por Flick (2009), os nomes dos participantes não foram divulgados e foi solicitada uma autorização formal por parte do IIF para publicação deste trabalho.

Com relação ao caso de estudo, trata-se do Instituto Internacional de Física (IIF), estabelecido na Universidade Federal do Rio Grande do Norte (UFRN) que foi concebido com o apoio estratégico do então Ministério da Ciência e Tecnologia (MCT). O Instituto é voltado puramente para a pesquisa, ensino e disseminação da física teórica, sua missão é "produzir, fomentar e promover a pesquisa em áreas de fronteiras da Física, garantindo a ampla difusão do conhecimento científico, a formação de pesquisadores em alto nível e a integração com outras áreas da ciência e da educação" (IIF, 2019). Sua visão é "ser uma instituição de prestígio internacional, resultado da excelência na produção científica e da promoção de eventos científicos integrados à pesquisa e à formação de pesquisadores de alta qualificação" (IIF, 2019). 
O IIF é responsável por impulsionar a internacionalização da física teórica produzida na UFRN por meio da produção científica de fronteira e de excelência, prezando pela valorização das pessoas, pelo comprometimento e pelas cooperações interinstitucionais. Desde sua inauguração, em maio de 2010, o IIF mantém um crescimento de cooperação em suas linhas de pesquisa com mais de 20 países, entre eles: Alemanha; Itália; Inglaterra; Suíça; Rússia; Canadá; Argentina; Chile; Estados Unidos; Reino Unido e França. O que resultou em mais de 400 artigos publicados, 366 seminários de pesquisa e 84 eventos internacionais que, alcançaram mais de 4000 pesquisadores e estudantes dos diversos países envolvidos. Atualmente, o IIF dispõe de acadêmico 45 cientistas (brasileiros e estrangeiros) divididos entre os conselhos consultivos nacional e internacional, além de 79 físicos pós-doutores, professores associados, pesquisadores e professores visitantes, distribuídos em 6 linhas de pesquisa.

Em 2018, houve a necessidade de submissão para a publicização do IIF como uma Organização Social (OS), nos termos da Lei Federal n. 9.637, de 18/05/1998. Lei pela qual o Poder Executivo poderá qualificar, como OS, pessoas jurídicas de direito privado sem fins lucrativos, cujas atividades sociais sejam dirigidas ao ensino, à pesquisa científica, ao desenvolvimento tecnológico, à proteção e preservação do meio ambiente, à cultura e à saúde, desde que atendidos os requisitos previstos nesse mesmo diploma.

Nesse sentido, foi demandado pelos órgãos regulamentadores a elaboração de um planejamento estratégico para quatro anos, o que foi realizado de acordo com o conceito de NBP apresentado por Veras (2017). A equipe foi formada pelo então vice-diretor, o gerente administrativo, um analista de sistemas, todos do IIF, uma consultora externa e um consultor sênior especialista no assunto.

Após a coleta dos dados, procedeu-se com a análise dos dados. Segundo Yin (2016), o processo de análise de dados qualitativos é composto por cinco fases: compilação, decomposição, recomposição, interpretação e conclusão. Neste estudo, buscou-se seguir essa orientação, realizando-se as devidas adaptações dadas as características da pesquisa. Primeiramente, todos os dados obtidos foram compilados, criando-se a base de dados que sustentaria a investigação. Após esse passo, foram atribuídos rótulos aos dados com o intuito de agrupar aqueles que representavam um mesmo tema. $\mathrm{Na}$ sequência buscou-se criar uma estrutura lógica para esses grupos que representasse o processo observado. Essa etapa foi fundamental para a análise, pois permitiu compreender a sequência dos passos e a importância dela para o processo como um todo. A quarta fase se iniciou praticamente sobreposta à anterior - entende-se que foi necessário um esforço analítico para a estruturação dos dados - e se prolongou quase até a finalização da subsequente. De modo geral, nesse momento buscouse estabelecer uma relação entre os materiais coletados e aquilo que foi observado no campo. Por fim, o esforço analítico foi direcionado para a extração de conclusões sobre o processo estudado que 
pudessem contribuir com o conhecimento sobre o processo de planejamento estratégico em negócios baseados em projetos.

De modo geral, entende-se que os procedimentos metodológicos adotados foram adequados ao propósito do estudo bem como aos recursos disponíveis para sua realização. Ressalta-se que as informações apresentadas na próxima seção foram orientadas pelos documentos levantados em campo e fundamentados pelas inferências obtidas a partir das atividades de observação realizadas.

\section{0 caso do Instituto Internacional de Física}

\subsection{Processo de elaboração do estratégico do IIF}

Para construção do planejamento estratégico foi utilizado um framework proposto por Veras (2017), que relaciona as ferramentas e técnicas utilizadas no processo de elaboração do planejamento estratégico e os resultados da aplicação dessas ferramentas. A Figura 4 apresenta esse framework.

\section{Figura 4}

Framework do processo de elaboração do planejamento estratégico

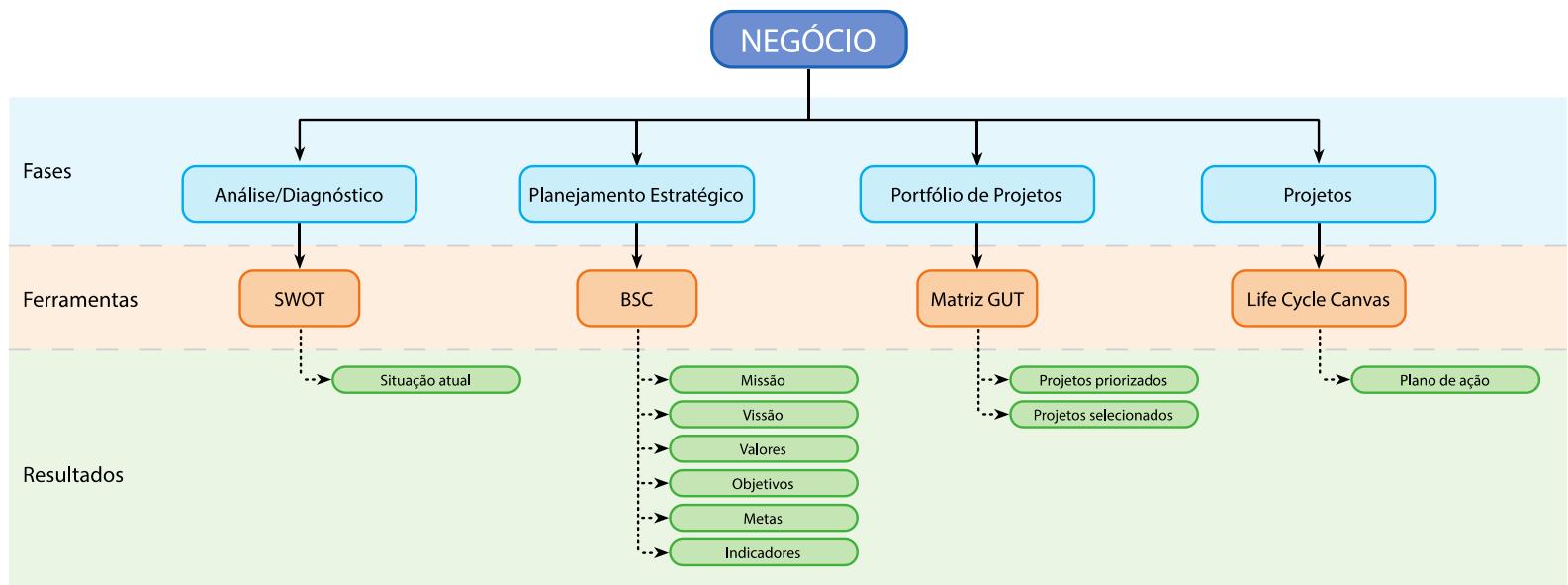

Fonte: Elaborado pelos autores a partir de Veras (2017).

Para a fase de análise e diagnóstico foi aplicada a matriz SWOT, acrônimo para Strengths (Forças), vantagens internas da organização em relação às concorrentes; Weaknesses (Fraquezas), desvantagens internas da organização em relação às concorrentes; Opportunities (Oportunidades), aspectos positivos com potencial de fazer crescer a vantagem competitiva da organização; Threats (Ameaças), aspectos negativos com potencial de comprometer a vantagem competitiva da organização. A Figura 5 mostra o resultado da aplicação da ferramenta. 
Figura 5

\section{Análise SWOT do IIF}

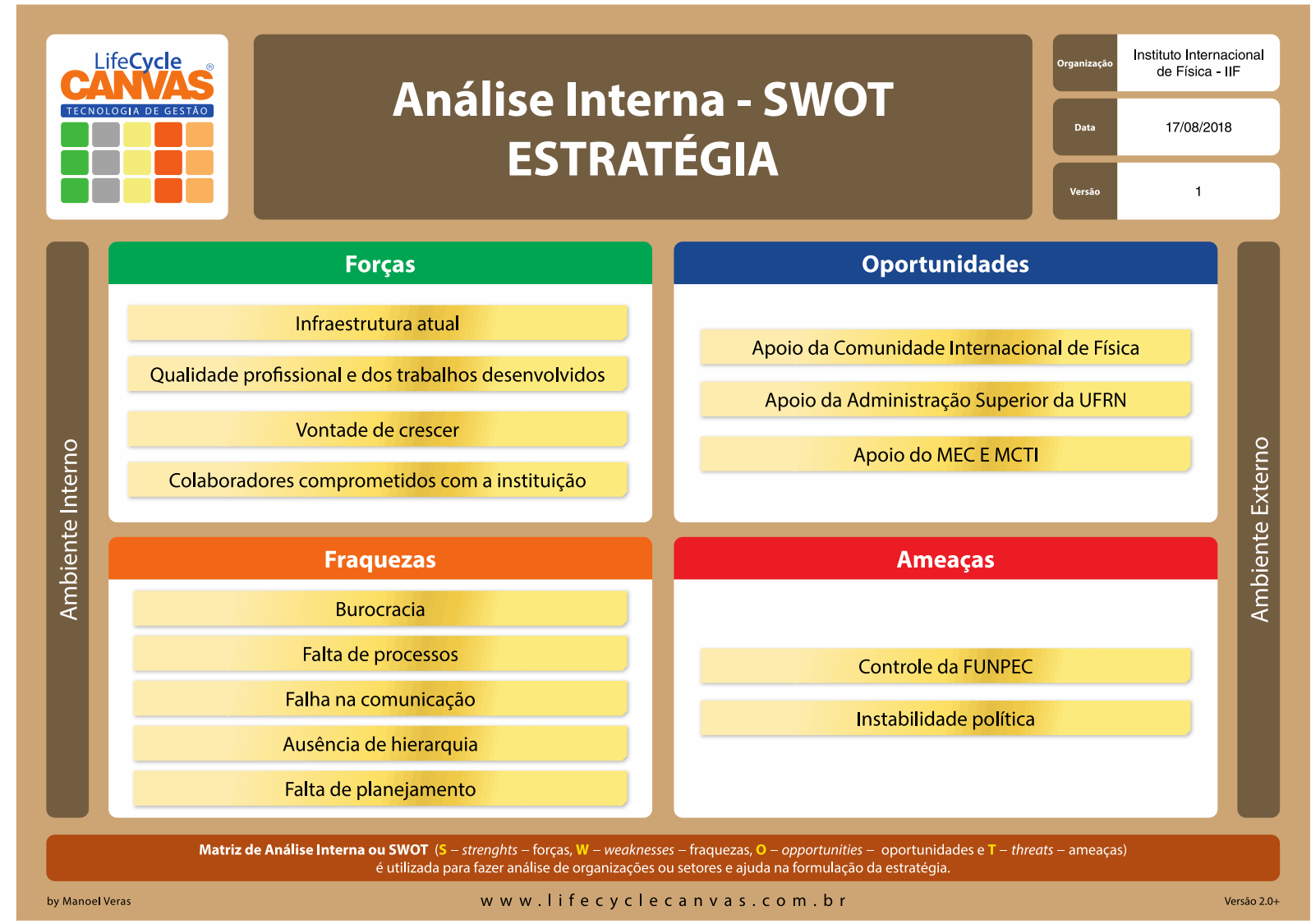

Fonte: dados da pesquisa (2019).

Após a análise dos ambientes interno e externo promovida pela matriz SWOT, foram definidos o negócio, a missão, a visão e os valores considerando uma perspectiva de quatro anos (2019-2022), ficando com as seguintes descrições:

- Negócio: Produção científica de fronteira e de excelência;

- Missão: Produzir, fomentar e promover a pesquisa em áreas de fronteira da Física, garantindo a ampla difusão do conhecimento científico, a formação de pesquisadores em alto nível e a integração com outras áreas da ciência e da educação;

- Visão: Ser uma instituição de prestígio internacional, resultado da excelência na produção científica, na promoção de eventos científicos integrados à pesquisa e à formação de pesquisadores de alta qualificação;

- Valores: Internacionalização, produção científica de fronteira, excelência, valorização das pessoas e comprometimento. 
O próximo passo foi a estruturação do planejamento estratégico, à luz do Balanced Scorecard (BSC). Destaca-se que as perspectivas clássicas do BSC (cliente, finanças, processos internos e aprendizado e crescimento) foram adaptadas de acordo com as escolhas estratégicas do IIF. Além disso, o BSC para instituições de caráter público deve refletir a estratégia em questão. A arquitetura do BSC com a perspectiva financeira no topo da hierarquia não serve para essas instituições. Numa perspectiva social ou governamental de BSC os recursos devem alicerçar as outras perspectivas (Veras, 2017). No âmbito de uma OS, como é o caso do IIF, é frequente a utilização da perspectiva orçamentária como base para as demais perspectivas do BSC.

Desse modo, as perspectivas do BSC do Instituto foram compostas por 6 seis grupos temáticos: Orçamento, Desenvolvimento Institucional, Pesquisa, Extensão, Intercâmbio e Resultados. Também foram definidos objetivos estratégicos para cada perspectiva criada, conforme apresentado no Mapa Estratégico da Figura 6. O Mapa Estratégico é a representação gráfica da estratégia construída com base no BSC em que são explicadas as relações de causa e efeito e os ativos intangíveis são relacionados aos outros ativos (Veras, 2017).

A partir dos dados obtidos nesta fase, foi possível a definição dos indicadores e metas para cada objetivo, como também a elaboração de projetos que visam o alcance desses objetivos. Esse processo alinha o planejamento estratégico com os projetos, visto que, cada projeto atenderá a um ou mais objetivos ou poderá um objetivo ser alcançado por mais de um projeto. 


\section{Mapa Estratégico do IIF}

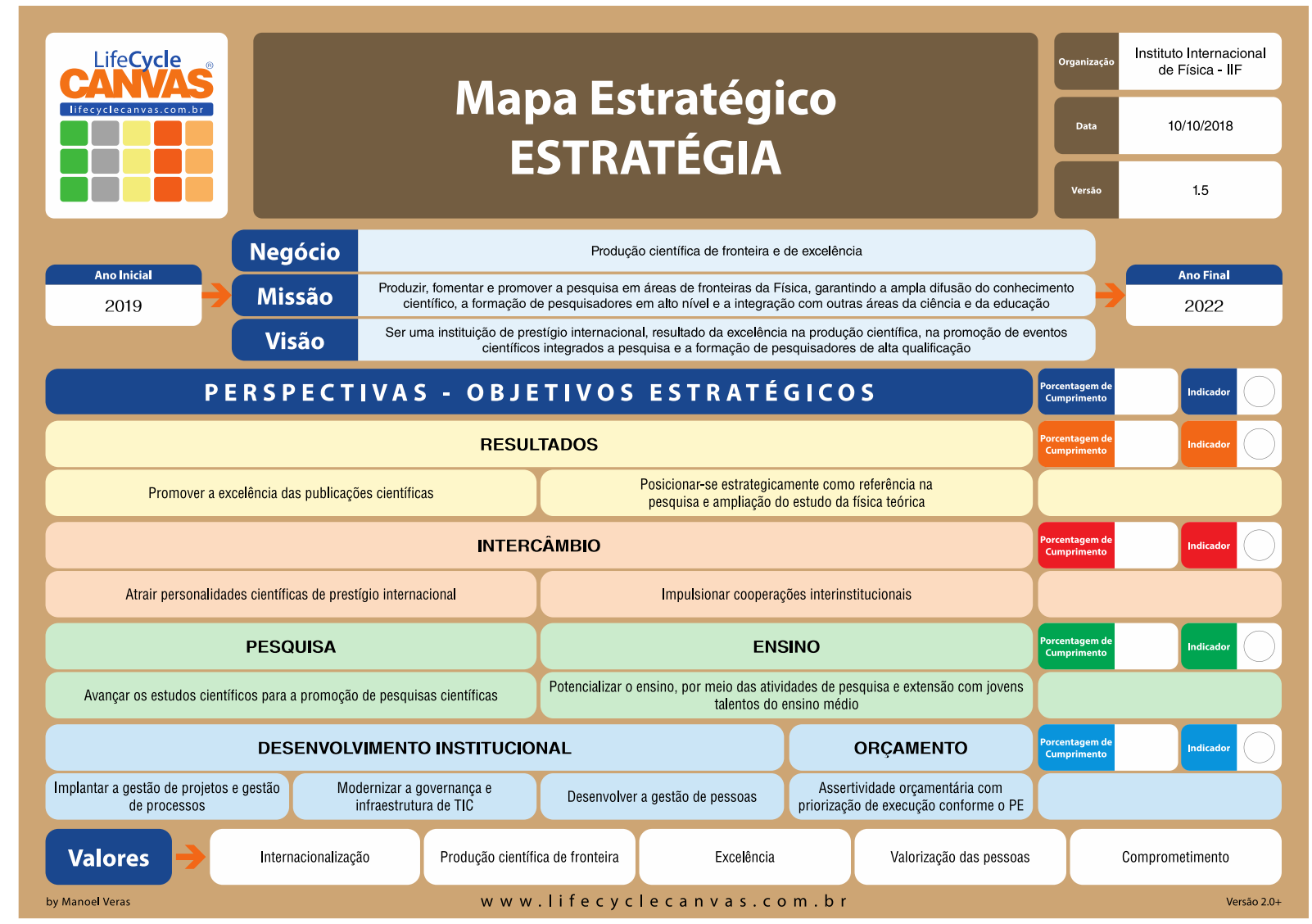

Fonte: Dados da pesquisa (2019)

Em seguida foram elaborados projetos, atendendo a necessidade dos objetivos estabelecidos. A Figura 7 detalha as relações entre os objetivos, indicadores, metas e projetos das perspectivas de desenvolvimento institucional e orçamento.

Na Figura 7 são apresentados os indicadores associados a cada objetivo estratégico, bem como a fórmula de cálculo dos indicadores e as metas previstas para os quatro anos. Ao lado esquerdo da figura tem-se os projetos relacionados a cada objetivo estratégico que, quando executados, darão vida à implementação do planejamento estratégico. 
Figura 7

Relacionamento dos objetivos, indicadores, metas e projetos

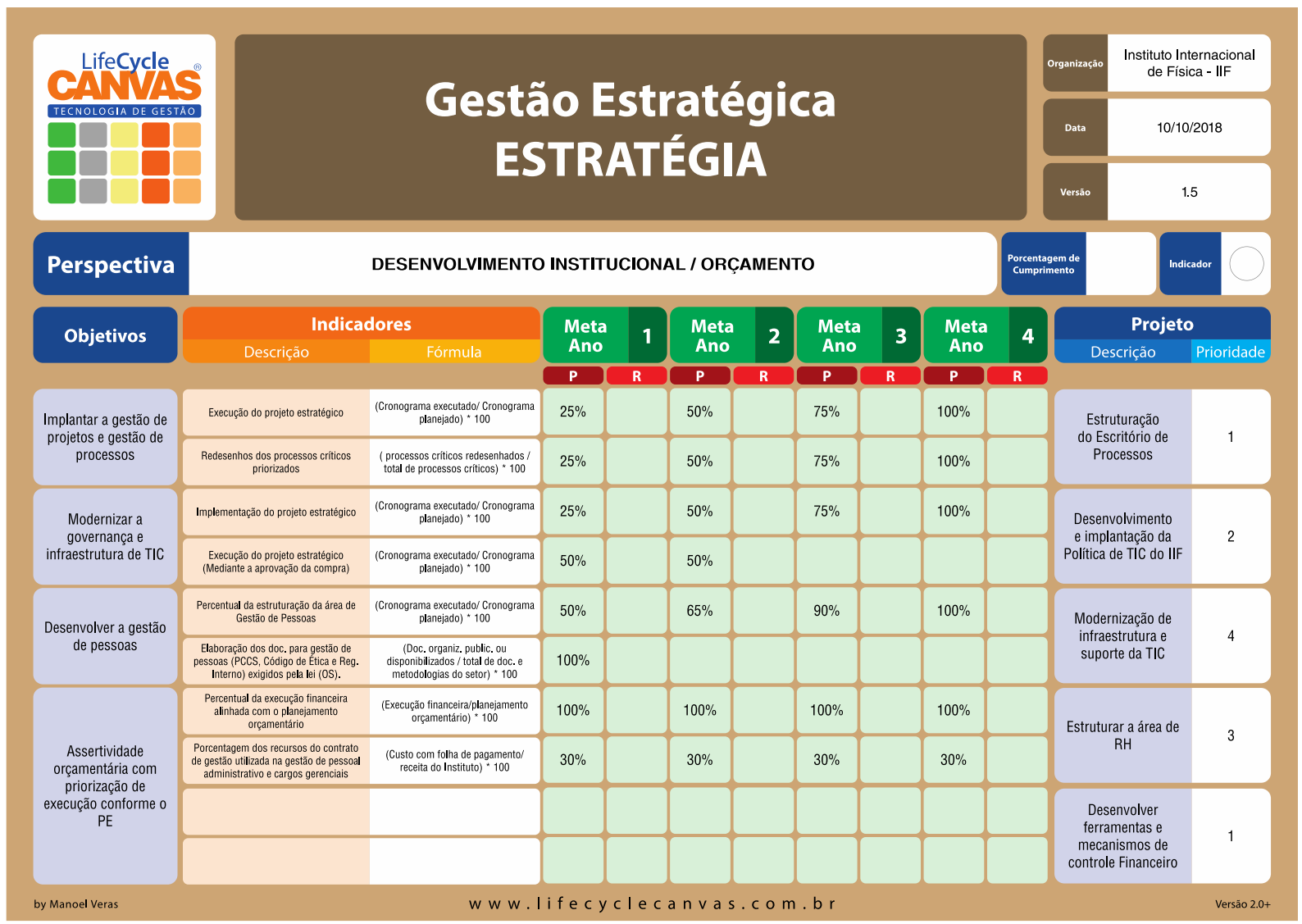

Fonte: Dados da pesquisa (2019).

Além dos projetos descritos na Figura 7, fizeram parte da priorização outros projetos e programas, todos vinculados aos objetivos estratégicos. O resultado é o produto de uma matriz GUT (Gravidade, Urgência e Tendência), utilizada durante para priorização dos projetos do portfólio.

Finalmente, cada projeto foi detalhado e elaborado seguindo a metodologia Life Cycle Canvas. A metodologia NBP possibilitou, de forma clara, delimitar os projetos a partir do desdobramento dos objetivos estratégicos e com critérios de priorização e seleção, o que aumenta a probabilidade de sucesso (Freitas, 2016; Veras, 2017). A Figura 8 mostra um dos projetos elaborados com a metodologia Life Cycle Canvas. 
Projeto Life Cycle Canvas - Estruturação do Escritório de Processos

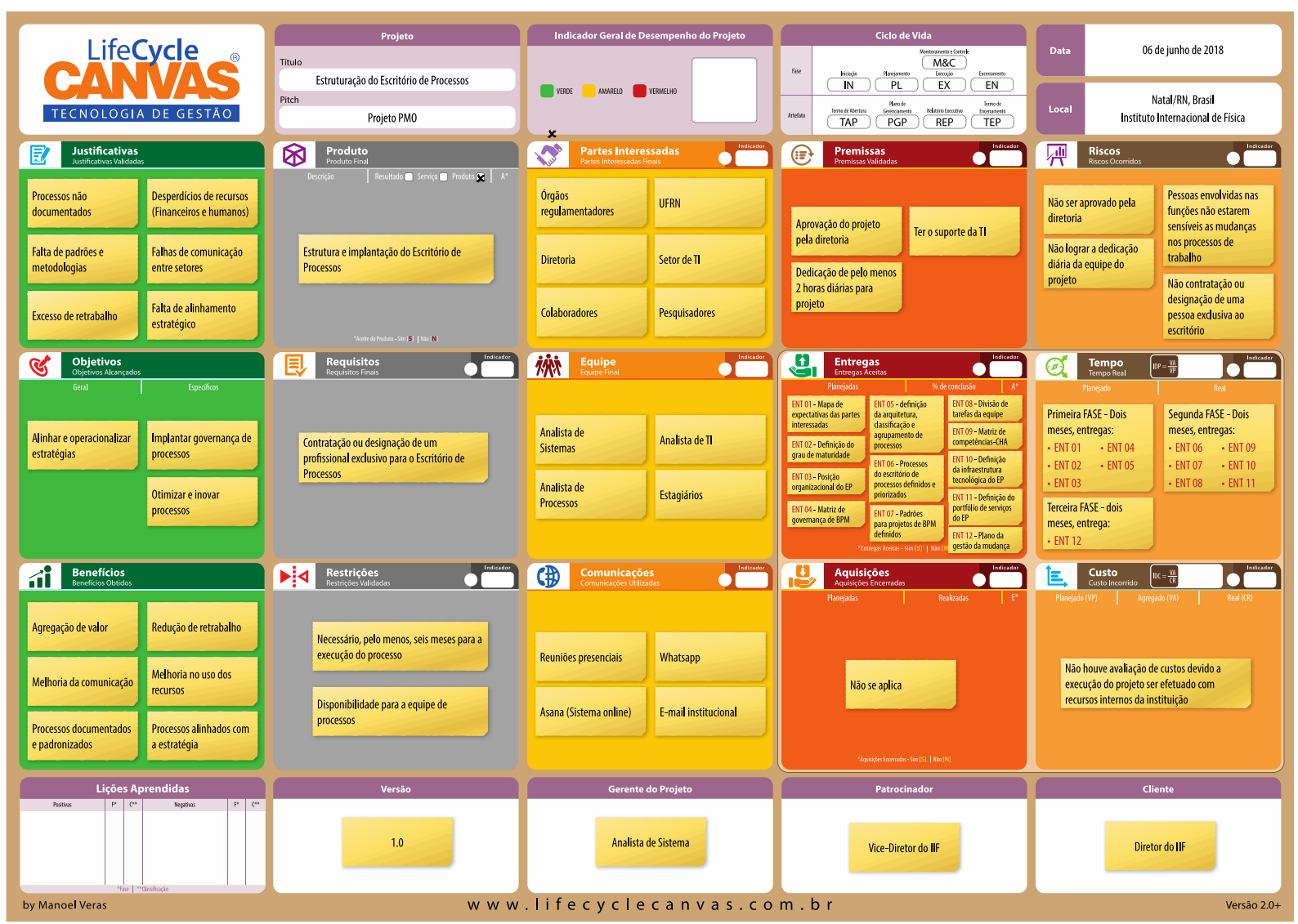

Fonte: Dados da pesquisa (2019).

Após apresentação do processo de elaboração do planejamento estratégico do IIF, a próxima seção apresenta uma discussão à luz da literatura.

\subsection{Discussão dos resultados}

A literatura aponta para uma forte relação de alinhamento do negócio com o desdobramento do plano estratégico em projetos (Alsudiri, Al-Karaghouli \& Eldabi,2013), resultado do uso de metodologias e conceitos abordados durante o processo de elaboração do planejamento (Lord, 1993; Cocks, 2010).

Essas iniciativas favorecem a aceitação pelos membros da organização, bem como por stakeholders relevantes para o processo, tornando mais factual a possibilidade de os projetos serem executados da forma como foram planejados. Nesse sentido, Serra e Kunk (2015) reforçaram que essas ações são apropriadas, pois incrementam a probabilidade de a estratégia organizacional ser implementada de forma bem-sucedida.

De modo geral, entende-se que a elaboração do planejamento estratégico do IIF baseada no 
conceito de NBP atendeu as orientações dos autores que versam sobre o tema, pois o processo apresentado permite visualizar a estratégia definida para a organização, bem como os projetos a serem executados para que os objetivos estratégicos sejam alcançados (Veras, 2017).

Além disso, observa-se que o estabelecimento de ações futuras, considerando o diagnóstico do ambiente mostrado na Figura 5, é um fator que contribui com a capacidade da organização alcançar as metas estabelecidas, dado que o processo de execução de uma estratégia organizacional é algo complexo e que normalmente ocorre em contextos dinâmicos e mutáveis (Dietrich \& Lehtonen, 2006).

Analisando o processo descrito à luz da gestão de portfólio, que é um tema relevante quando se trata da elaboração da estratégia a partir de projetos (Guedes et al., 2011; Meskedahl, 2010), verificase que as ações com o intuito de priorizar os projetos ocorrem na metodologia adotada por meio da aplicação da Matriz GUT. Essas atividades visam maximizar o valor dos projetos para o contexto organizacional em razão de sua contribuição para o planejamento estratégico organizacional.

Tais aspectos vão ao encontro dos achados de Cocks (2010) e Silva, Nascimento e Araújo (2017) no tocante as contribuições da gestão de projetos à implementação da estratégia. A utilização do NBP apresentado neste trabalho corrobora essas contribuições no tocante a fornecer uma estrutura para monitoramento e controle, e para comunicação e feedback ao processo de implementação da estratégia. Além disso, a utilização do NBP contribuiu para o alinhamento e a integração da gestão de projetos com o processo de formulação da estratégia, o que é visto na literatura como um fator crítico de sucesso (Lord, 1993; Cocks, 2010; Alsudiri, Al-Karaghouli \& Eldabi, 2013).

\section{Considerações finais}

Este trabalho se dedicou a apresentar o processo de elaboração de planejamento estratégico do IIF, o qual teve sua construção inteiramente baseada no conceito de Negócio Baseado em Projetos. Enquanto resultado principal, verificou-se que a utilização do NBP permitiu a formulação de projetos a partir do desdobramento dos objetivos estratégicos, com critérios de priorização e seleção. Além disso, observou-se que a metodologia utilizada também proporcionou engajamento das partes envolvidas na elaboração do planejamento.

Destaca-se que o esforço empregado pela equipe para a elaboração de um planejamento estratégico alinhado ao negócio e a projetos foi bem-sucedido do ponto de vista das expectativas da instituição. As técnicas e ferramentas utilizadas, bem como a metodologia definida e a elaboração de forma compartilhada e participativa, por meio de reuniões e entrevistas, geraram indícios positivos para a efetividade do planejamento, além de poderem ser utilizadas como base para outras organizações.

Desse modo, destaca-se que o presente trabalho colabora para o enriquecimento dos estudos na área, uma vez que mostra o desdobramento de um planejamento estratégico por projetos como 
forma de promover sua implementação. Assunto este pouco abordado pela literatura, mas que demonstrou bons resultados quando aplicado na prática.

O trabalho apresenta como limitação a escassez de estudos que abordam o conceito de negócio NBP, o que teria permitido uma revisão teórica mais ampla, e a existência de poucos trabalhos anteriores que analisam o processo de elaboração de um planejamento estratégico com base no conceito de negócio baseado em projetos, o que teria possibilitado uma análise mais fundamentada. Além dessa, pontua-se que, em razão do horizonte temporal no qual esta pesquisa foi realizada, não foi possível acompanhar a implementação do planejamento cujo processo de elaboração foi apresentado neste trabalho. Acredita-se que este acompanhamento potencializaria os resultados apresentados, no entanto, considerando o propósito descritivo da investigação, ressalta-se que o estudo alcançou o objetivo proposto.

Todavia tais dificuldades referendam a contribuição do presente trabalho para a literatura, bem como destacam a relevância da pesquisa ora apresentada. Como sugestão para pesquisas futuras, indica-se o acompanhamento do planejamento em sua integridade, ou seja, da elaboração até seu encerramento. O que permitiria maior assertividade nos resultados alcançados, além de possibilitar um aprofundamento maior do assunto para o futuro. Apesar desta análise está embasada na literatura e confrontada na realidade, é possível o surgimento de interpretações divergentes, mesmo que a pesquisa tenha validado os resultados alcançados.

\section{Referências}

Alsudiri, T., Al-Karaghouli, W., \& Eldabi, T. (2013). Alignment of large project management process to business strategy: A review and conceptual framework. Journal of Enterprise Information Management, 26(5), 596-615. https://doi.org/10.1108/JEIM-07-2013-0050

Anselmo, J. L., \& Maximiano, A. C. A. (2011). Administração estratégica em organizacões orientadas para projetos. Revista de Gestão e Projetos-GeP, 2(2), 03-25. http://dx.doi.org/10.5585/10.5585

Cocks, G. (2010). Emerging concepts for implementing strategy. The TQM Journal, 22(3), 260-266. https://doi.org/10.1108/17542731011035505

Creswell, J. W. (2014). Investigação Qualitativa e Projeto de Pesquisa-: Escolhendo entre Cinco Abordagens. Porto Alegre: Penso Editora.

Dietrich, P., \& Lehtonen, P. (2005). Successful management of strategic intentions through multiple projects: reflections from empirical study. International Journal of Project Management, 23 (5), 386-391. https://doi.org/10.1016/j.ijproman.2005.03.002

Flick, U. (2009). Introdução à pesquisa qualitativa. Porto Alegre: Artmed. 
Freitas, C. A. (2016). Gestão Estratégica por meio de Projetos, Programas e Portfólio (Série Estratégia em Projetos, Programas e Portfólio). Rio de Janeiro: Brasport.

Guedes, R. M., Fonseca, F., Carvalho, K. L. de, Maximiano, A. C. A., \& Gonçalves, M. A. (2011). Alinhamento do portfólio de projetos à estratégia das organizações: um estudo exploratório quantitativo. Revista Economia \& Gestão, 11 (27), 67-93. https://doi.org/10.5752/P.19846606.2011v11n27p67

Hill, C., \& Jones, G. (2009). Theory of Strategic management with Cases. [sI]: Cengage South-Western, 1032 p. ISBN 978-1-4390-3560-3.

Hrebiniak, L. G. (2006). Obstacles to effective strategy implementation. Organizational Dynamics, 35 (1), 12 - 31. https://doi.org/10.1016/j.orgdyn.2005.12.001

IIF. (2019). Instituto Internacional de Física (UFRN). https://www.iip.ufrn.br/aboutdetail

Jamieson, A., \& Morris, P. W. (2007). Moving from corporate strategy to project strategy. In The Wiley Guide to Project, Program, and Portfolio Management (p. 34-62). John Wiley \& Sons Hoboken, NJ.

Lord, M. A. (1993). Implementing strategy through project management. Long Range Planning, 26(1), 76-85. https://doi.org/10.1016/0024-6301(93)90236-9

Melton, T., Iles-Smith, P., \& Yates, J. (2008). Project benefits management: linking your project to the Business. London: Butterworth-Heinemann.

Meskendahl, S. (2010). The influence of business strategy on project portfolio management and its success: a conceptual framework. International Journal of Project Management, 28 (8), 807817. https://doi.org/10.1016/j.ijproman.2010.06.007

Mintzberg, H. (2004). Ascensão e queda do planejamento estratégico. Porto Alegre: Bookman Editora.

Musawir, Ata ul, Serra, C. E. M., Zwikael, O., \& Ali, I. (2017). Project governance, benefit management, and project success: towards a framework for supporting organizational strategy implementation. International Journal of Project Management, 35 (8), 1658-1672. https://doi.org/10.1016/j.ijproman.2017.07.007

Project Management Institute (PMI). (2017). A guide to the project management body of knowledge PMBok. 6. ed. Pennsylvania: Project Management Institute.

Porter, M. E. (1996). What is strategy. Published November.

Serra, C. E. M., \& Kunc, M. (2015). Benefits realisation management and its influence on project success and on the execution of business strategies. International Journal of Project Management, 33(1), 53-66. https://doi.org/10.1016/j.ijproman.2014.03.011

Saunders, M., Lewis, P., \& Thornhill, A., (2016). Research methods for business students (seventh ed.). London: Prentice Hall.

Silva, R. R., Nascimento, T. H. C. R., \& Araújo, A. G. (2017). A contribuição do gerenciamento de projetos na implementação de estratégia: uma revisão sistemática da literatura. In: VI Simpósio Internacional de Gestão de Projetos (SINGEP), São Paulo, SP, Brasil. 
Silva Filho, A. M., Silva, R. R., Silva, D. C., \& Medeiros, M. F. M. (2018). O processo empreendedor: associando o Business Model Canvas (BMC) ao Life Cycle Canvas (LCC). Exacta, 16(4), 35-44. https://doi.org/10.5585/Exacta.v16n4.7991

Vargas, R. V. (2005). Gerenciamento de Projetos (6a edição). Rio de Janeiro: Brasport.

Veras, M. (2017). Negócio baseado em projetos. Rio de Janeiro: Brasport.

Veras, M., \& Campelo, B. (2016). Life Cycle Canvas: gestão dinâmica de projetos. Revista Mundo PM, 70.

Yin, R. K. (2016). Pesquisa qualitativa do início ao fim. Porto Alegre: Penso Editora. 